\title{
Development of nomograms related to inflammatory biomarkers to estimate the prognosis of bladder cancer after radical cystectomy
}

\author{
Wentao Zhang ${ }^{1,2 \#}$, Fuhan Yang ${ }^{1,2 \#}$, Aimaitiaji Kadier ${ }^{1,2 \#}$, Yifan Chen ${ }^{1,2}$, Yang Yu ${ }^{1,2}$, Junfeng Zhang ${ }^{1,2}$, \\ Jiang Geng ${ }^{1,2}$, Yang Yan $^{1,2}$, Wei $\mathbf{L i}^{1,2}$, Xudong Yao ${ }^{1,2} \wedge$ \\ ${ }^{1}$ Department of Urology, Shanghai Tenth People's Hospital, Tongji University School of Medicine, Shanghai, China; ${ }^{2}$ Institute of Urinary Oncology, \\ School of Medicine, Tongji University, Shanghai, China \\ Contributions: (I) Conception and design: X Yao; (II) Administrative support: W Li, Y Yan; (III) Provision of study materials or patients: W Zhang, A \\ Kadier; (IV) Collection and assembly of data: F Yang, Y Chen, Y Yu; (V) Data analysis and interpretation: J Zhang, J Geng; (VI) Manuscript writing: \\ All authors; (VII) Final approval of manuscript: All authors. \\ \#These authors contributed equally to this work. \\ Correspondence to: Prof. Xudong Yao; Prof. Wei Li; Prof. Yang Yan. Department of Urology, Shanghai Tenth People's Hospital, Tongji University, \\ Shanghai 200072, China. Email: yaoxudong1967@163.com; liweitongji@163.com; 13564368328@163.com.
}

Background: Bladder cancer is one of the most common carcinomas and it brings about huge social economic burden. There is not a reliable way to predict the prognosis of bladder patients. We develop the nomogram to predict the prognosis of bladder cancer patients.

Methods: A total of 127 bladder cancer patients after radical cystectomy were studied retrospectively. Their clinicopathological data were collected for statistical analysis.

Results: The level of albumin/globulin ratio (AGR), C-reactive protein/albumin ratio (CAR), neutrophil to lymphocyte ratio (NLR) and platelet to lymphocyte ratio (PLR) associated with pathological and hematological parameters like $\mathrm{T}$ stage and hemoglobin. Furthermore, the AGR was associated with overall survival (OS) and CAR, NLR, and PLR were associated with both OS and progression-free survival (PFS) $(\mathrm{P}<0.05)$. The multivariate analysis revealed that tobacco smoking, tumor $T$ stage, $M$ stage, NLR, CAR, and AGR were all independent predictors for OS of patients and tobacco smoking, tumor T stage, NLR, CAR, and AGR were independent predictors for PFS of patients. In addition, AGR, CAR, and NLR, as well as, the clinicopathological parameters in the development of nomograms with a C index of 0.901 (95\% CI: $0.505-$ 1.269) for OS, and 0.807 (95\% CI: 0.755-0.858) for PFS. The nomograms were able to provide a prognosis of the OS with the area under the curve $(\mathrm{AUC})=0.86$. Further, tests assessed the PFS with the AUC $=0.84$.

Conclusions: This study demonstrates that the nomograms of the inflammatory biomarkers were able to predict prognosis of bladder cancer patients after radical cystectomy.

Keywords: Nomogram; bladder cancer; inflammatory biomarkers; prognosis

Submitted Jul 16, 2021. Accepted for publication Sep 01, 2021.

doi: 10.21037/atm-21-4097

View this article at: https://dx.doi.org/10.21037/atm-21-4097

^ ORCID: 0000-0001-7234-3940. 


\section{Introduction}

Bladder cancer is a significant global health problem, with 429,800 newly diagnosed cases and 165,100 cancer-related deaths worldwide in 2012 (1). In China, 80,000 new cases and 32,000 cancer-related deaths were reported in 2015. To date, approximately $75 \%$ of the diagnosed bladder cancer cases are non-muscle invasive bladder cancer (NMIBC), whereas the remaining $25 \%$ of patients have muscleinvasive bladder cancer (MIBC). Unfortunately, patients diagnosed with MIBC often have a poorer prognosis than patients with NMIBC.

Patients who have undergone radical cystectomy have shown better prognosis than patients without surgery (2). However, there are no reliable biomarkers for the prediction of bladder cancer and post-cystectomy treatment response. Therefore, the identification and evaluation of biomarkers is essential for the risk stratification of patients, assessment of treatment decisions, and prediction of bladder cancer prognosis. Previous studies reported that bladder cancer development and progression were closely related to the host inflammatory and immune responses. The progression and metastasis of tumor cells occur through various mechanisms such as the inflammatory response (3). During the inflammatory response, a number of neutrophils and platelets, as well as acute phase proteins such as C-reactive protein and albumin, are altered. Many of these inflammatory indicators show potential as markers for the clinical assessment of cancer prognosis. For example, the albumin/globulin ratio (AGR), C-reactive protein/ albumin ratio (CAR), neutrophil to lymphocyte ratio (NLR), and platelet to lymphocyte ratio (PLR) have been used to predict the prognosis of bladder cancer patients $(4,5)$. However, conflicting reports on the effectiveness of these measured parameters requires further investigation to determine their predictive potential. Therefore, we developed nomograms to assess the usefulness of these parameters for predicting bladder cancer prognosis.

We present the following article in accordance with the TRIPOD reporting checklist (available at https://dx.doi. org/10.21037/atm-21-4097).

\section{Methods}

\section{Patients and data collection}

In this study, we retrospectively included 150 bladder cancer patients who received medical care between January 2005 and December 2017 at Shanghai Tenth People's Hospital (Shanghai, China). Patients were selected based on the following criteria: (I) histopathological diagnosis of bladder cancer including MIBC and recurrence of high-grade T1 (T1HG) bladder cancer; (II) laboratory tests were collected 3 days before surgery, including serum levels of albumin, C-reactive protein, and neutrophil count, among others; and (III) complete clinical and follow-up data was collected. Patients with a history of cardiovascular and cerebrovascular disease and type 2 diabetes were also included in the study. Patients who received neoadjuvant/adjuvant chemotherapy or suffered from other inflammatory conditions were excluded from the study. Therefore, a total of 127 patients could be used to construct the nomograms.

The following clinicopathological data were collected from patient medical records: age, gender, smoking status, tumor size, cancer history, laboratory tests (urinary cytology, cystoscopy, serum creatinine, ultrasonography, and computed tomography or magnetic resonance imaging), and pathological findings (tumor histology, staging, grading, and lymph nodes). Tumor histology was classified as urothelial carcinoma or squamous cell carcinoma. Pathologists assessed the pathological grade and stage of the tumor according to the 2004 WHO classification system and the tumor, lymph node, metastasis (TNM) staging system. The AGR was calculated as albumin divided by globulin, and the CAR was calculated as C-reactive protein divided by albumin. The NLR and PLR were calculated as platelets, and the absolute count of neutrophils was divided by the absolute count of lymphocytes.

Patients were followed up by 2 experienced non-surgical physicians. Cystoscopy and urine cytology were performed every 3 months within 2 years after surgery, every 6 months for the next 3 years, and then annually. Abdominal CT examinations were performed every 6 months after surgery for 2 years, and once a year thereafter. All results were obtained through outpatient visits or telephone interviews. All patients were followed up regularly until March 2018. The overall survival (OS) was defined as the duration between the first tumor diagnosis and death, while the progression-free survival (PFS) was defined as the time between the first tumor diagnosis and disease progression after treatment.

The study was approved by the Ethics Committee of the Shanghai Tenth People's Hospital (SHSYIEC-4.1/19-120/01), and all participants provided written informed consent before registration. The study is also in 
line with the Helsinki Declaration (as revised in 2013).

\section{Statistical analysis}

SPSS v24.0 (SPSS Inc., Chicago, IL, USA) was used for all statistical analyses. The median and interquartile range (IQR) were used to evaluate continuous variables, while frequency and scale were used to classify variables. Receiver operating characteristic (ROC) curves were used to determine the best cut-off values for the AGR, CAR, NLR, and PLR. Kaplan-Meier curves and the log rank test were used to predict OS and PFS after stratified sampling of the AGR, CAR, NLR, and PLR. Univariate and multivariate Cox regression analyses were performed to calculate the corresponding hazard ratio (HR) and 95\% confidence interval (CI). Nomograms and time-dependent ROCs were established using R (http://www.R-project. org) and EmpowerStats software (www.empowerstats.com, The $\mathrm{X} \& \mathrm{Y}$ solutions, Inc., Boston MA). A P value $<0.05$ was considered statistically significant.

\section{Results}

\section{Characteristics of patients}

We retrospectively analyzed a cohort of 127 bladder cancer patients after radical cystectomy. The clinicopathological data showed that there were 110 males and 17 females with a median age of 66 years old (ranging between 29 and 87 years). Based on clinical records, 61 patients had smoked tobacco and 66 never smoked tobacco. Of all bladder cancer patients, 17 patients had low-grade bladder cancer while 110 had high-grade bladder cancer. Of the high-grade bladder cancer cases, 48 cases $(37.8 \%)$ were stage T1, 26 (20.5\%) were stage T2, 29 (22.8\%) were stage T3, and 24 (18.9\%) were stage T4. The mean body mass index (BMI) of these patients was 23.47. The pathology reports of 108 patients showed urothelial carcinoma, whereas the other 19 patients had squamous cell carcinoma.

\section{Identification of the inflammatory biomarkers and their cut-off values}

In order to more accurately determine the cut-off values for the assessed biomarkers, we plotted data using ROC curves to identify the pre-surgery values for the AGR, CAR, NLR, and PLR. It was ascertained that $A G R=1.55$, $\mathrm{CAR}=0.165, \mathrm{NLR}=3.73$, and PLR $=124.4$ were the cut- off values for each parameter and were identified as the best prognostic predictors for patients after radical cystectomy (Figure 1). Specifically, the area under the curve (AUC) of the AGR was 0.81 (95\% CI: 0.74-0.88), with a sensitivity of 0.84 and a specificity of $0.66(\mathrm{P}<0.001)$. The AUC of the CAR was 0.76 (95\% CI: $0.68-0.85)$, with a sensitivity of 0.67 and a specificity of $0.83(\mathrm{P}<0.001)$. The AUC of the NLR was 0.62 (95\% CI: $0.53-0.72$ ), with a sensitivity of 0.47 and a specificity of $0.81(\mathrm{P}<0.012)$. The AUC of the PLR was 0.62 (95\% CI: $0.52-0.72$ ), with a sensitivity of 0.72 and a specificity of $0.45(\mathrm{P}<0.015)$. Based on these cut-off values, we were able to separate these 127 patients into the following groups: low AGR group with $73(57.5 \%$; <1.55), high AGR group with 54 (42.5\%; $\geq 1.55)$; low CAR group with $75(59.1 \%$; <0.16), high CAR group with 52 (40.9\%; $\geq 0.16)$; low NLR group with $76(59.8 \%$; $<3.73)$, high NLR group with $51(40.2 \% ; \geq 3.73)$; low PLR group with 45 (35.4\%; <106), high PLR group with $82(64.6 \% ; \geq 106)$.

\section{Association of these inflammatory biomarkers with the clinicopathological features of patients}

The identified inflammatory biomarkers were then linked with patient clinicopathological features. We found that a high AGR level was associated with tobacco smoking, tumor size, $\mathrm{T}$ stage, tumor-infiltrating neutrophils, and levels of serum albumin and hemoglobin $(\mathrm{P}<0.05)$. The CAR biomarker correlated with tobacco smoking, $\mathrm{T}$ stage, $\mathrm{N}$ stage, tumor-infiltrating neutrophils, and serum levels of albumin and hemoglobin $(\mathrm{P}<0.05)$. NLR levels were linked with tobacco smoking, tumor size, $\mathrm{T}$ stage, $\mathrm{N}$ stage, and levels of serum albumin, neutrophils, and hemoglobin $(\mathrm{P}<0.05)$. Moreover, a high PLR level corresponded with high BMI, tobacco smoking, and serum levels of hemoglobin $(\mathrm{P}<0.05$; Table 1).

Furthermore, we plotted the Kaplan-Meier curves for OS and PFS stratified by these parameters and discovered that AGR levels predicted the OS of these patients, while levels of the CAR, NLR, and PLR forecasted both OS and $\mathrm{PFS}(\mathrm{P}<0.05$; Figure 1).

\section{Univariate and multivariate analyses of $O S$ and PFS}

Univariate and multivariate analyses were employed to predict independent factors of OS and PFS. Our univariate analysis indicated that tobacco smoking, tumor size, T stage, $\mathrm{N}$ stage, $\mathrm{M}$ stage, NLR, PLR, CAR, AGR, and albumin levels were all significantly linked to the OS of patients 

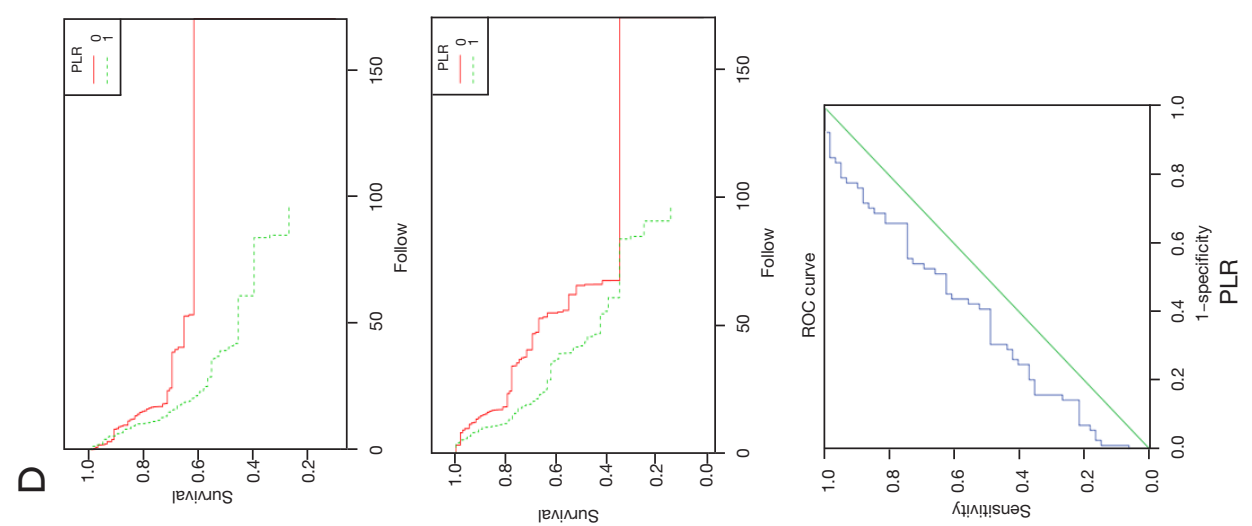

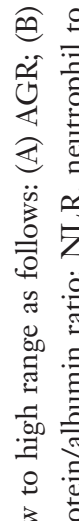
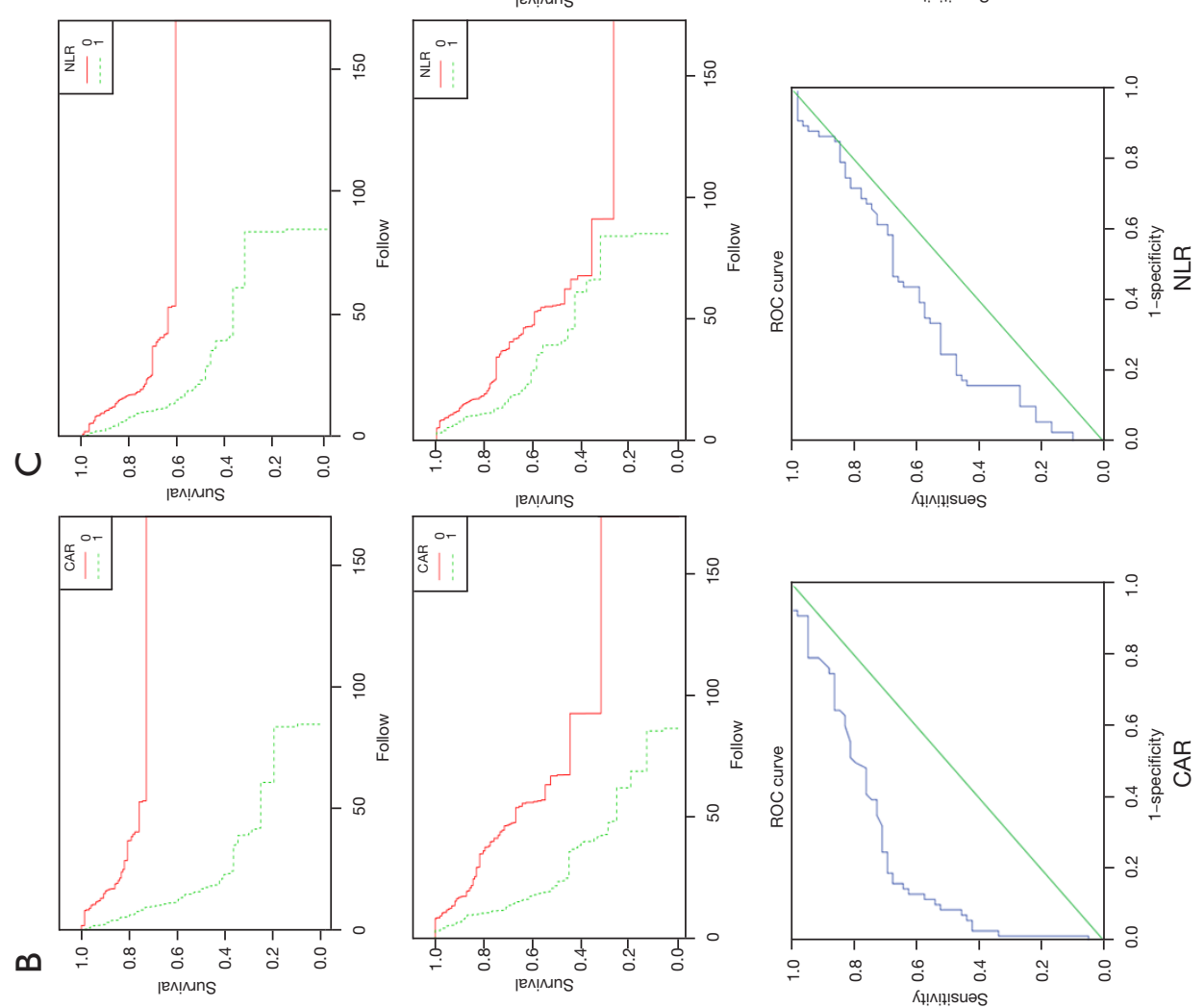

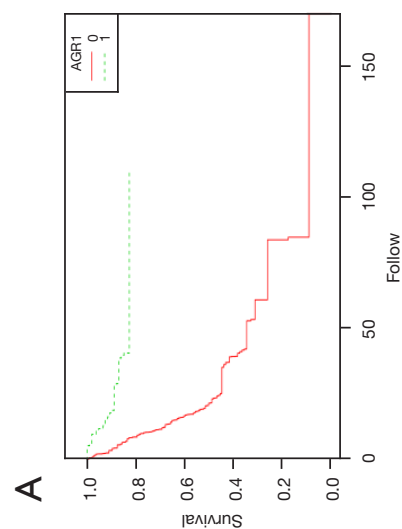

\&

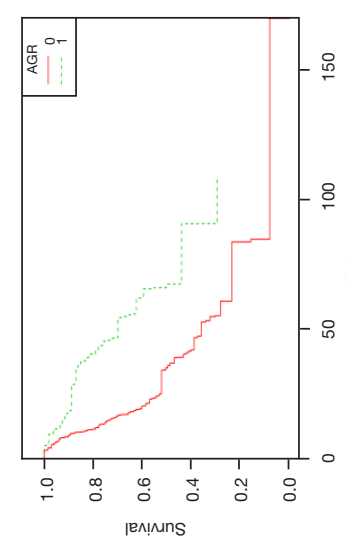

自

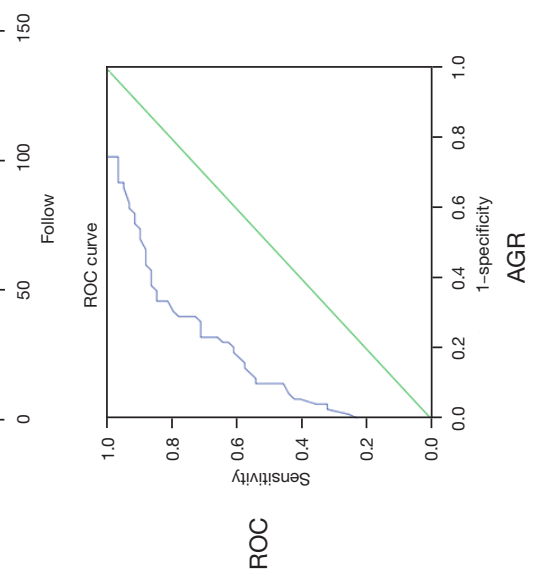

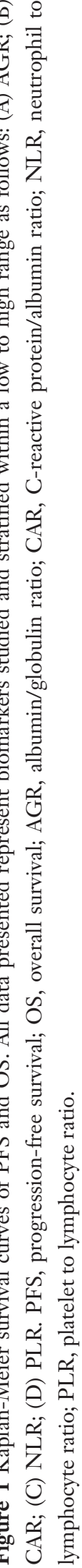




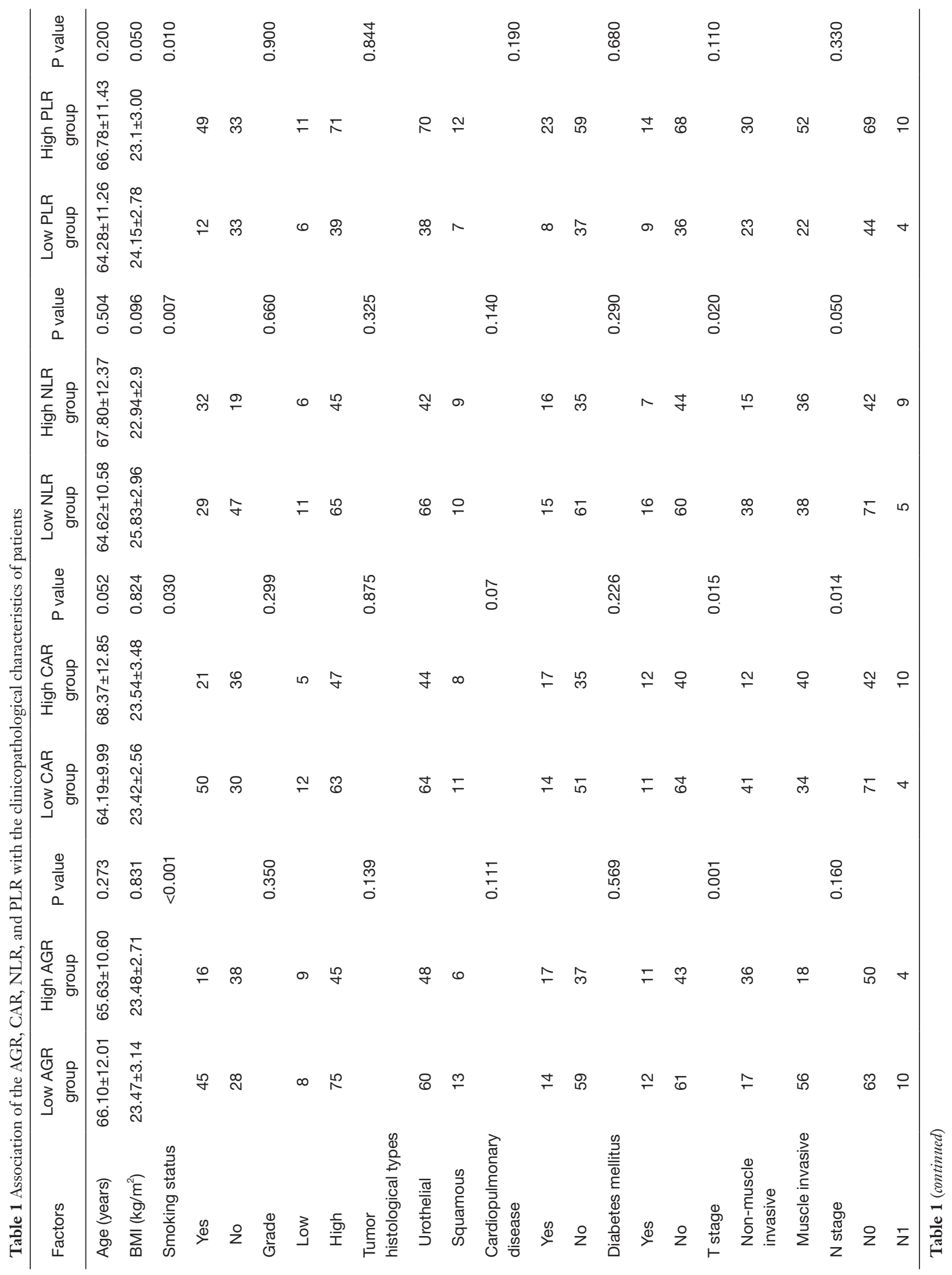




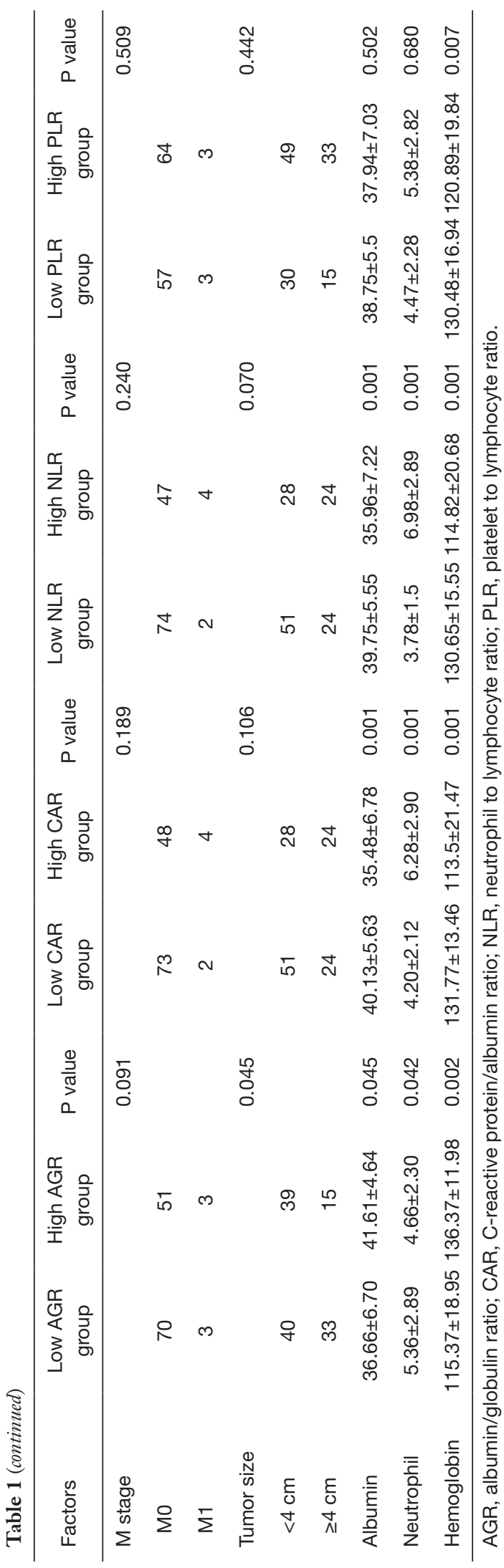

$(\mathrm{P}<0.05$; Table 2). Fewer clinicopathological features, such as tobacco smoking, tumor diameter, T stage, $\mathrm{N}$ stage, NLR, AGR, CAR, and levels of albumin and hemoglobin, were associated with $\mathrm{PFS}(\mathrm{P}<0.05$; Table 3). Our multivariate analysis revealed that tobacco smoking, $\mathrm{T}$ stage, $\mathrm{M}$ stage, NLR, CAR, and AGR were independent predictors for the OS of patients, while tobacco smoking, T stage, NLR, $\mathrm{CAR}$, and AGR were independent predictors for the PFS of patients $(\mathrm{P}<0.05$; Table 2 and Table 3).

\section{Development of the nomogram models of these inflammatory biomarkers for bladder cancer}

Based on the significant independent factors of PFS and OS and from the data collected on the NLR, PLR, CAR, and AGR, we developed OS and PFS predictive nomogram models for bladder cancer patients using multivariate Cox regression analysis. We determined these scores based on the intersection of the vertical line and the point axis associated with each variable, while the total risk scores were summations of each variable score. Thus, these total scores established predictive measures of OS and PFS for each patient. Moreover, we revealed that the Harrell's C-index of the nomogram was 0.901 (95\% CI: 0.50-1.26) for OS and 0.81 (95\% CI: 0.75-0.85) for PFS (Figure 2). In addition, we analyzed the performance of the nomogram by time-dependent ROC curves compared with the TNM staging system, and for OS, we observed an AUC of 0.86, sensitivity of 0.96 , and specificity of 0.63 , while for PFS, the AUC was 0.84 , the sensitivity was 0.83 , and the specificity was 0.67 (Figure 3).

\section{Discussion}

Previous studies have assessed different inflammatory biomarkers including the AGR, CAR, NLR, and PLR to predict bladder cancer progression and prognosis. However, the data reported are inconsistent. Variations in laboratory test results and differences in the optimal cutoff values are likely to affect the data analysis and reported data accuracy. In the current study, we determined the optimal cut-off values of the AGR, CAR, NLR, and PLR in the prediction of bladder cancer prognosis using ROC curves. Our multivariate Cox analysis identified the AGR, CAR, and NLR as independent prognostic predictors. Through analyzing pre-surgery clinicopathological features and inflammatory biomarkers (AGR, CAR, and NLR), we have successfully developed 2 useful nomograms to 
Table 2 Prognostic factors for OS in bladder cancer patients after radical cystectomy

\begin{tabular}{|c|c|c|c|c|c|c|}
\hline Factors & \multicolumn{3}{|c|}{ Univariate analysis } & \multicolumn{3}{|c|}{ Multivariate analysis } \\
\hline Sex & 0.67 & $0.28-1.56$ & 0.35 & & & \\
\hline Age & 1.91 & $1.10-3.34$ & 0.21 & & & \\
\hline BMI & 0.89 & $0.53-1.49$ & 0.67 & & & \\
\hline Cardiopulmonary disease & 1.17 & $0.65-2.07$ & 0.59 & & & \\
\hline Diabetes mellitus & 1.36 & $0.73-2.53$ & 0.32 & & & \\
\hline Tumor size & 1.84 & $1.10-3.09$ & 0.02 & 1.54 & $0.90-2.64$ & 0.11 \\
\hline T stage & 7.04 & $3.32-14.94$ & 0 & 3.72 & $1.66-8.32$ & 0.001 \\
\hline Tumor histological types & 1.35 & $0.83-2.19$ & 0.223 & & & \\
\hline Grade & 1.58 & $0.68-3.68$ & 0.28 & & & \\
\hline AGR & 0.15 & $0.075-0.31$ & 0 & 0.25 & $0.11-0.55$ & 0.001 \\
\hline NLR & 2.79 & $1.65-4.69$ & 0.001 & 2.11 & $1.05-4.24$ & 0.019 \\
\hline PLR & 2.04 & $1.12-3.73$ & 0.019 & & & \\
\hline CAR & 4.94 & $2.85-8.57$ & 0 & 2.31 & $1.14-4.71$ & 0.02 \\
\hline Albumin & 0.59 & $0.34-1.02$ & 0.06 & & & \\
\hline Hemoglobin & 0.31 & $0.17-0.55$ & 0 & 0.78 & $0.40-1.51$ & 0.46 \\
\hline
\end{tabular}

AGR, albumin/globulin ratio; CAR, C-reactive protein/albumin ratio; NLR, neutrophil to lymphocyte ratio; PLR, platelet to lymphocyte ratio.

predict the OS and PFS of bladder cancer patients after radical cystectomy. Most importantly, we were able to use these nomograms to predict OS and PFS in each individual patient, contributing to the potential for personalized therapy.

Recently, nomograms have been used to predict the probability of clinical outcomes in a variety of cancer patients. Several studies have investigated clinical and pathological variables in the prognostic prediction of bladder cancer after radical surgery. Bochner et al. developed a nomogram including age, gender, operative time, pathological tumor stage, grading, and regional lymph node status to predict recurrence after radical surgery for bladder cancer (6). Yamada et al. used the data of tumor number, shape, grade, and intravesical instillation to construct a nomogram and used it to predict recurrencefree survival (7). In these studies, only the clinical and pathological data of the patients were included. In our study, we included patients' inflammation index, immune status, and clinicopathological data to make the results more convincing. We also noticed that Riester et al. included certain novel genes (such as FN1, NNMT, POSTN, and SMAD6) in their nomogram, which may make the predictions more accurate (8). However, such genetic testing has a higher financial burden. In China, measuring inflammation indicators such as the CAR and NLR may be less expensive and more affordable. Using these clinically readily available indicators to initially predict the prognosis of patients with bladder cancer may promote the individualized treatment of patients by doctors.

In recent years, the use of inflammation-related markers as predictive measures for determining the prognosis of cancer patients has received increasing attention $(9,10)$. For bladder cancer in particular, several previous studies have confirmed the association of inflammation-related markers with the prognosis of patients. For example, 
Table 3 Prognostic factors for PFS in bladder cancer patients after radical cystectomy

\begin{tabular}{|c|c|c|c|c|c|c|}
\hline Factors & \multicolumn{3}{|c|}{ Univariate analysis } & \multicolumn{3}{|c|}{ Multivariate analysis } \\
\hline Sex & 0.53 & $0.22-1.23$ & 0.14 & & & \\
\hline Age & 1.51 & $0.91-2.53$ & 0.11 & & & \\
\hline BMI & 1.20 & $0.73-1.97$ & 0.45 & & & \\
\hline Cardiopulmonary disease & 1.11 & $0.64-1.94$ & 0.702 & & & \\
\hline Diabetes mellitus & 1.50 & $0.82-2.72$ & 0.18 & & & \\
\hline Tumor size & 1.73 & $1.03-2.89$ & 0.036 & 1.35 & $0.79-2.31$ & 0.26 \\
\hline T stage & 3.94 & $2.21-7.01$ & $<0.001$ & 4.36 & $1.93-9.85$ & 0.001 \\
\hline Tumor histological types & 1.43 & $0.89-2.32$ & 0.14 & & & \\
\hline Grade & 1.87 & $0.80-4.33$ & 0.14 & & & \\
\hline AGR & 0.42 & $0.24-0.71$ & 0.001 & 0.27 & $0.12-0.62$ & 0.002 \\
\hline CAR & 3.16 & $1.92-5.22$ & 0.001 & 2.30 & $1.19-4.42$ & 0.013 \\
\hline NLR & 1.70 & $1.20-2.42$ & 0.003 & 1.00 & $0.66-1.52$ & 0.01 \\
\hline PLR & 1.51 & $1.03-2.20$ & 0.031 & 0.84 & $0.44-1.63$ & 0.62 \\
\hline Albumin & 0.52 & $0.31-0.89$ & 0.019 & 0.91 & $0.44-1.89$ & 0.81 \\
\hline Hemoglobin & 0.41 & $0.24-0.69$ & 0.001 & 0.77 & $0.37-1.57$ & 0.47 \\
\hline
\end{tabular}

AGR, albumin/globulin ratio; CAR, C-reactive protein/albumin ratio; NLR, neutrophil to lymphocyte ratio; PLR, platelet to lymphocyte ratio.

Rajwa et al. evaluated the prognostic efficacy of the PLR, NLR, derived neutrophil-lymphocyte ratio (dNLR), and lymphocyte-monocyte ratio (LMR) in bladder cancer patients (11). Cheah et al. established that high CD14 expression in bladder cancer cells increased recruitment of more inflammatory factors, polarizing monocytes and macrophages to alter their immunosuppressive characteristics and promoting tumor cell growth (12). In this regard, the inflammation responses in bladder cancer demonstrate the systemic host immune status and reveal the complexity of the damage to immune surveillance caused by these cancer cells (13). Here, we assessed the values of these inflammation factors to evaluate and predict the prognosis of bladder cancer patients after radical cystectomy.

Furthermore, the value of the AGR is equal to the ratio of albumin/globulin, whereby serum albumin is primarily produced by the liver (14). Studies showed that a decrease in the levels of blood albumin led to an increase in systemic malnutrition and inflammation (15), while there was a close interaction between the blood albumin level and tumorigenesis (16). Conversely, globulin is a proinflammatory protein with complex components, including C-reactive protein (CRP), complement components, and immunoglobulin (17). Globulin's involvement in chronic inflammation was observed via elevated levels associated with inflammatory responses and immunosuppression (18). High serum levels of Globulin (GLB) resulted in the poor prognosis of several cancers $(19,20)$. An increase in CRP has been reported in many cancer cases, which reflects the inflammatory and immune response against tumor cells of the human body (21). In the current study, we first revealed the association of the CAR with bladder cancer as an independent prognostic predictor.

Additionally, previous studies have demonstrated that 

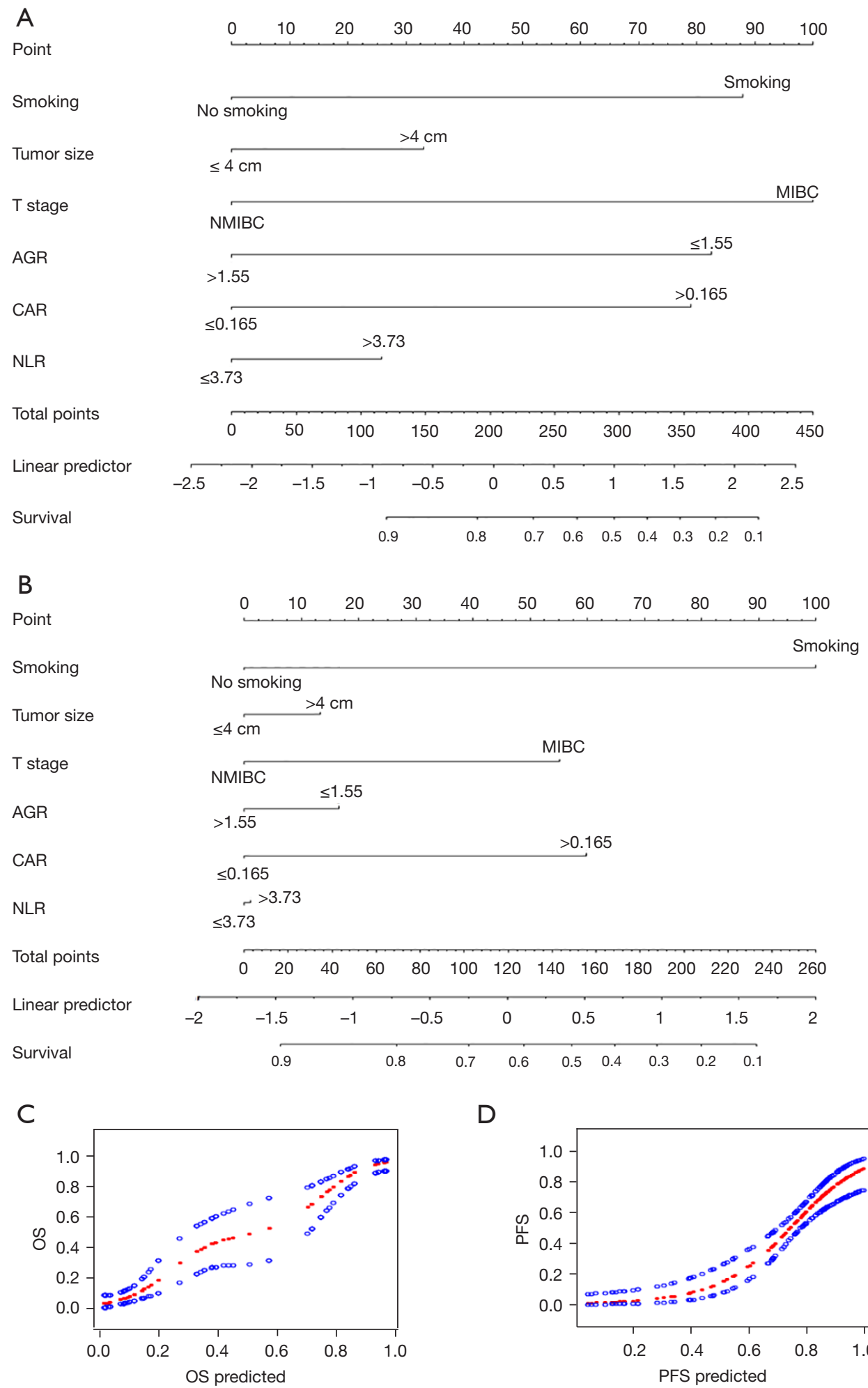

$\mathrm{D}$

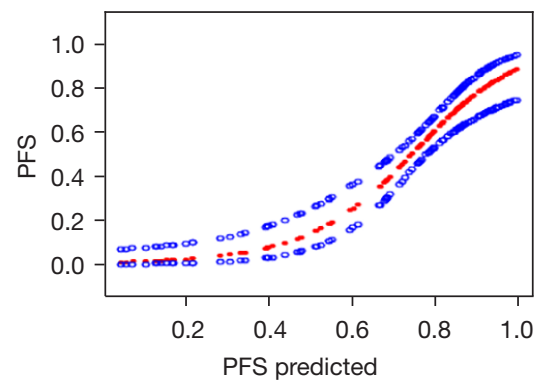

Figure 2 Nomogram models of inflammatory biomarkers. Nomograms convey prognostic models stratified by the clinicopathological characteristics and pretreatment inflammatory biomarkers for (A) OS prediction; (B) PFS prediction; (C) the Harrell's C-index to predict OS; (D) the Harrell's C-index to predict PFS. OS, overall survival; PFS, progression-free survival. 

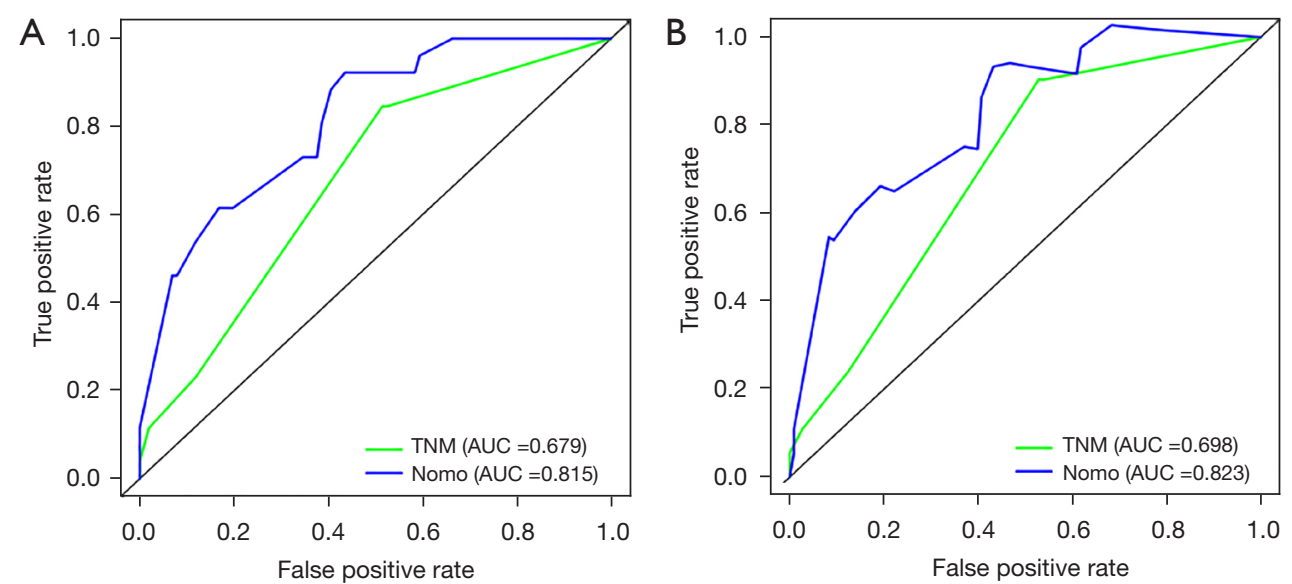

Figure 3 ROC curves compared with the TNM staging system. Time-dependent ROC curves by nomogram and TNM staging system for (A) OS prediction; and (B) PFS prediction. ROC, receiver operating characteristic; OS, overall survival; PFS, progression-free survival.

the NLR and PLR are indicators of bladder cancer $(22,23)$. The level of NLR reflects the human body's immune response affiliated with cancer immunosurveillance, immunoediting, and immunosubversion (24). The upregulation of NLR indicated the relative increase in neutrophils and decrease in lymphocytes, leading to the promotion of tumor inflammation and an increase in the risk of cancer metastasis and recurrence (25). Meanwhile, neutrophils secrete IL-6, vascular endothelial growth factor (VEGF), and transforming growth factor beta (TGF- $\beta$ ) in the tumor microenvironment, resulting in the induction of lymphocyte apoptosis and immunosuppression (26). Albisinni et al. also suggest NLR and HPR seem closely related to the RFS, CSS and OS in bladder cancer (27). Platelets also play an important role in the recruitment and regulation of monocytes and granulocytes into tumor lesions (13), suggesting that platelet functionality may be critical for the production of tumor-associated macrophages and tumor-associated neutrophils (28). However, the PLR as an independent predictor of prognosis remains controversial, and many reports have shown that PLR does not have a significant ability to predict prognosis. Giakoustidis et al. (29) investigated the role of the NLR and PLR in the prognosis of 127 patients undergoing pancreatic ductal adenocarcinoma resection. They demonstrated the disadvantage of PLR as a prognostic factor (30). In our study, we also found that the PLR was not significantly associated with prognosis. However, further research is still needed to assess PLR as a prognostic factor in bladder cancer.

Smoking is known as one of the most significant risk factors for bladder cancer $(31,32)$. Studies have also shown that smoking affects the prognosis of patients with bladder cancer $(33,34)$. In this study, all inflammatory biomarkers were associated with smoking in patients. Smoking can induce systemic inflammation by increasing oxidative stress. Smoking also enhances the activation of redoxsensitive transcription factors such as nuclear factor-kappa $\mathrm{B}(\mathrm{NF}-\mathrm{\kappa B})$ and activator protein-1 (AP-1), which induce IL-8, IL-6, and tumor necrosis factor- $\alpha$ (TNF- $\alpha$ ), leading to systemic inflammation $(30,35)$. Therefore, smoking affects the body's immune regulation through these factors. Thus, compared with non-smoking patients, smoking can promote the development of tumors.

Most of the pathological diagnosis of bladder cancer is urothelial carcinoma, and a few are squamous cell carcinoma or other pathological types. For squamous cell carcinoma, in Egypt and other countries, the incidence of bladder cancer is closely related to schistosomiasis infection, and inflammation plays an important role in it (36). In our cohort, no significant differences in inflammatory markers between squamous cell carcinoma and urothelial carcinoma were observed. Severin Rodler et al. showed that changes in platelets and hemoglobin were not observed as independent risk factors in the bladder squamous cell carcinoma cohort (37). This may require more pathological data of squamous cell carcinoma for further research.

However, our current study does have some limitations. For example, our study is a single-center retrospective cohort study. Thus, we are not convinced of the efficacy of this predictive model, particularly when evaluating different ethnic groups. In order to enhance the model, further 
research is required through larger multicenter prospective cohort studies. Moreover, the data of this group of patients may not represent other bladder cancer patients who have refused surgical intervention for various reasons.

\section{Conclusions}

In summary, our current data suggest that preoperative inflammatory biomarkers (AGR, NLR, and CAR) are independent prognostic predictors for the PFS and OS of bladder cancer patients after radical cystectomy. The nomograms based on these inflammatory biomarkers more accurately and practically predicted the OS and PFS of bladder cancer patients after radical cystectomy.

\section{Acknowledgments}

Funding: This work was supported in part by a grant from the National Natural Science Foundation of China (\#81472389); the Shanghai Science Committee Foundation (\#19411967700); National Natural Science Foundation of China, Youth Project (\#81602469); Shanghai Pujiang Talent Program (\#20PJ1412400) and General Project of Shanghai Natural Science Foundation of China (\#20ZR1443000).

\section{Footnote}

Reporting Checklist: The authors have completed the TRIPOD reporting checklist. Available at https://dx.doi. org/10.21037/atm-21-4097

Data Sharing Statement: Available at https://dx.doi. org/10.21037/atm-21-4097

Conflicts of Interest: All authors have completed the ICMJE uniform disclosure form (available at https://dx.doi. org/10.21037/atm-21-4097). The authors have no conflicts of interest to declare.

Ethical Statement: The authors are accountable for all aspects of the work in ensuring that questions related to the accuracy or integrity of any part of the work are appropriately investigated and resolved. The study was approved by the Ethics Committee of the Shanghai Tenth People's Hospital, and all participants provided written informed consent before registration. The study is also in line with the Helsinki Declaration (as revised in 2013).
Open Access Statement: This is an Open Access article distributed in accordance with the Creative Commons Attribution-NonCommercial-NoDerivs 4.0 International License (CC BY-NC-ND 4.0), which permits the noncommercial replication and distribution of the article with the strict proviso that no changes or edits are made and the original work is properly cited (including links to both the formal publication through the relevant DOI and the license). See: https://creativecommons.org/licenses/by-nc-nd/4.0/.

\section{References}

1. Torre LA, Bray F, Siegel RL, et al. Global cancer statistics, 2012. CA Cancer J Clin 2015;65:87-108.

2. Lawrentschuk N, Colombo R, Hakenberg OW, et al. Prevention and management of complications following radical cystectomy for bladder cancer. Eur Urol 2010;57:983-1001.

3. Schepisi G, Santoni M, Massari F, et al. Urothelial Cancer: Inflammatory Mediators and Implications for Immunotherapy. BioDrugs 2016;30:263-73.

4. Niwa N, Matsumoto K, Ide H, et al. Prognostic Value of Pretreatment Albumin-to-Globulin Ratio in Patients With Non-Muscle-Invasive Bladder Cancer. Clin Genitourin Cancer 2018;16:e655-61.

5. Liu J, Dai Y, Zhou F, et al. The prognostic role of preoperative serum albumin/globulin ratio in patients with bladder urothelial carcinoma undergoing radical cystectomy. Urol Oncol 2016;34:484.e1-8.

6. International Bladder Cancer Nomogram Consortium; Bochner BH, Kattan MW, et al. Postoperative nomogram predicting risk of recurrence after radical cystectomy for bladder cancer. J Clin Oncol 2006;24:3967-72.

7. Yamada T, Tsuchiya K, Kato S, et al. A pretreatment nomogram predicting recurrence- and progression-free survival for nonmuscle invasive bladder cancer in Japanese patients. Int J Clin Oncol 2010;15:271-9.

8. Riester M, Taylor JM, Feifer A, et al. Combination of a novel gene expression signature with a clinical nomogram improves the prediction of survival in high-risk bladder cancer. Clin Cancer Res 2012;18:1323-33.

9. Ryan AM, Power DG, Daly L, et al. Cancer-associated malnutrition, cachexia and sarcopenia: the skeleton in the hospital closet 40 years later. Proc Nutr Soc 2016;75:199-211.

10. Eftekhari R, de Lima SG, Liu Y, et al. Microenvironment proteinases, proteinase-activated receptor regulation, 
cancer and inflammation. Biol Chem 2018;399:1023-39.

11. Rajwa P, Życzkowski M, Paradysz A, et al. Evaluation of the prognostic value of LMR, PLR, NLR, and dNLR in urothelial bladder cancer patients treated with radical cystectomy. Eur Rev Med Pharmacol Sci 2018;22:3027-37.

12. Cheah MT, Chen JY, Sahoo D, et al. CD14-expressing cancer cells establish the inflammatory and proliferative tumor microenvironment in bladder cancer. Proc Natl Acad Sci U S A 2015;112:4725-30.

13. Kim J, Bae JS. Tumor-Associated Macrophages and Neutrophils in Tumor Microenvironment. Mediators Inflamm 2016;2016:6058147.

14. Hoogenboezem EN, Duvall CL. Harnessing albumin as a carrier for cancer therapies. Adv Drug Deliv Rev 2018;130:73-89.

15. Laky B, Janda M, Cleghorn G, et al. Comparison of different nutritional assessments and bodycomposition measurements in detecting malnutrition among gynecologic cancer patients. Am J Clin Nutr 2008;87:1678-85.

16. Wang N, Liu JY, Li X, et al. Pretreatment serum albumin/ globulin ratio as a prognostic biomarker in metastatic prostate cancer patients treated with maximal androgen blockade. Asian J Androl 2018;21:56-61.

17. Chen J, Zhou Y, Xu Y, et al. Low pretreatment serum globulin may predict favorable prognosis for gastric cancer patients. Tumour Biol 2016;37:3905-11.

18. Sawada N, Iwasaki M, Inoue $M$, et al. Plasma testosterone and sex hormone-binding globulin concentrations and the risk of prostate cancer among Japanese men: a nested casecontrol study. Cancer Sci 2010;101:2652-7.

19. Shimizu T, Ishizuka M, Shibuya N, et al. Preoperative globulin-to-albumin ratio predicts outcome after curative resection in patients with gastric cancer. Ann Gastroenterol Surg 2018;2:367-75.

20. Li X, Qin S, Sun X, et al. Prognostic Significance of Albumin-Globulin Score in Patients with Operable Non-Small-Cell Lung Cancer. Ann Surg Oncol 2018;25:3647-59.

21. Black S, Kushner I, Samols D. C-reactive Protein. J Biol Chem 2004;279:48487-90.

22. Morizawa Y, Miyake M, Shimada K, et al. Correlation of Immune Cells and Cytokines in the Tumor Microenvironment with Elevated Neutrophil-ToLymphocyte Ratio in Blood: An Analysis of MuscleInvasive Bladder Cancer. Cancer Invest 2018;36:395-405.

23. Schulz GB, Grimm T, Buchner A, et al. Prognostic Value of the Preoperative Platelet-to-leukocyte Ratio for
Oncologic Outcomes in Patients Undergoing Radical Cystectomy for Bladder Cancer. Clin Genitourin Cancer 2017;15:e915-21.

24. Block MS, Markovic SN. The Tumor/Immune Interface: Clinical Evidence of Cancer Immunosurveillance, Immunoediting and Immunosubversion. American Journal of Immunology 2009;5:29-49.

25. Capone M, Giannarelli D, Mallardo D, et al. Baseline neutrophil-to-lymphocyte ratio (NLR) and derived NLR could predict overall survival in patients with advanced melanoma treated with nivolumab. J Immunother Cancer 2018;6:74.

26. Maeda K, Malykhin A, Teague-Weber BN, et al. Interleukin-6 aborts lymphopoiesis and elevates production of myeloid cells in systemic lupus erythematosus-prone B6.Sle1.Yaa animals. Blood 2009;113:4534-40.

27. Albisinni S, Pretot D, Al Hajj Obeid W, et al. The impact of neutrophil-to-lymphocyte, platelet-tolymphocyte and haemoglobin-to-platelet ratio on localised renal cell carcinoma oncologic outcomes. Prog Urol 2019;29:423-31.

28. Gros A, Ollivier V, Ho-Tin-Noé B. Platelets in inflammation: regulation of leukocyte activities and vascular repair. Front Immunol 2015;5:678.

29. Giakoustidis A, Neofytou K, Costa Neves M, et al. Identifying the role of neutrophil-to-lymphocyte ratio and platelets-to-lymphocyte ratio as prognostic markers in patients undergoing resection of pancreatic ductal adenocarcinoma. Ann Hepatobiliary Pancreat Surg 2018;22:197-207.

30. Hirota K, Matsui M, Iwata S, et al. AP-1 transcriptional activity is regulated by a direct association between thioredoxin and Ref-1. Proc Natl Acad Sci U S A 1997;94:3633-8.

31. Freedman ND, Silverman DT, Hollenbeck AR, et al. Association between smoking and risk of bladder cancer among men and women. JAMA 2011;306:737-45.

32. Dowdy D. Tobacco smoking and bladder cancer. JAMA 2011;306:2216-7; author reply 2217.

33. Hou L, Hong X, Dai M, et al. Association of smoking status with prognosis in bladder cancer: A meta-analysis. Oncotarget 2017;8:1278-89.

34. van Osch FH, Jochems SH, van Schooten FJ, et al. Significant Role of Lifetime Cigarette Smoking in Worsening Bladder Cancer and Upper Tract Urothelial Carcinoma Prognosis: A Meta-Analysis. J Urol 2016;195:872-9.

35. Rahman I, MacNee W. Role of transcription factors in 
inflammatory lung diseases. Thorax 1998;53:601-12.

36. Moschini M, D'Andrea D, Korn S, et al. Characteristics and clinical significance of histological variants of bladder cancer. Nat Rev Urol 2017;14:651-68.

37. Rodler S, Buchner A, Ledderose ST, et al. Prognostic

Cite this article as: Zhang W, Yang F, Kadier A, Chen Y, Yu Y, Zhang J, Geng J, Yan Y, Li W, Yao X. Development of nomograms related to inflammatory biomarkers to estimate the prognosis of bladder cancer after radical cystectomy. Ann Transl Med 2021;9(18):1440. doi: 10.21037/atm-21-4097 value of pretreatment inflammatory markers in variant histologies of the bladder: is inflammation linked to survival after radical cystectomy? World J Urol 2021;39:2537-43. 